\title{
Improvements in air quality: whose lungs benefit?
}

\author{
Ulrike Gehring ${ }^{1}$ and Gerard H. Koppelman (10) ${ }^{2,3}$
}

\begin{abstract}
Affiliations: ${ }^{1}$ Institute for Risk Assessment Sciences, Utrecht University, Utrecht, The Netherlands. ${ }^{2}$ University of Groningen, University Medical Center Groningen, Beatrix Children's Hospital, Dept of Pediatric Pulmonology and Pediatric Allergology, Groningen, The Netherlands. ${ }^{3}$ University of Groningen, University Medical Center Groningen, Groningen Research Institute for Asthma and COPD (GRIAC), Groningen, The Netherlands.
\end{abstract}

Correspondence: Ulrike Gehring, Utrecht University, Institute for Risk Assessment Sciences, P0 Box 80178, 3508 TD Utrecht, The Netherlands. E-mail: u.gehringquu.nl

@ERSpublications

The benefits of improved air quality extend across the life span into the elderly population http://ow.ly/Pi9q30o6M8o

Cite this article as: Gehring U, Koppelman GH. Improvements in air quality: whose lungs benefit? Eur Respir J 2019; 53: 1900365 [https://doi.org/10.1183/13993003.00365-2019].

Exposure to ambient air pollution is a major contributor to the global burden of disease, and is associated with increased risk of mortality and morbidity, in particular from pulmonary and cardiovascular diseases $[1,2]$. The health effects of air pollution have been intensively studied during the past decades. Acute effects have been demonstrated by short-term studies linking day-to-day variation in air pollution to day-to-day variation in health; chronic effects have been shown in long-term cohort studies following subjects with different levels of exposure over time [3].

Lung function is an objective marker of respiratory health and a predictor of cardiorespiratory morbidity and mortality [4]. It has been linked to air pollution in many cross-sectional and prospective cohort studies. The findings of these studies provide strong evidence for the adverse effects of long-term air pollution exposure on the lung function and lung function growth of children [5-8]. The reported effects of air pollution on lung function are generally small on the population level, in the order of a few percent $[5,7]$. Nevertheless, the public health impact is considerable due to the widespread nature of the exposure and the fact that small shifts in lung function at the population level result in significant increases in the percentage of subjects with lung function levels below clinical thresholds [9-11]. Moreover, lung function tracks throughout life, which means that the maximum attained lung function in early adulthood likely will be suboptimal in those with a low lung function in childhood, which puts them at an increased risk of developing chronic obstructive pulmonary disease (COPD) in adulthood [12]. Fewer studies have been performed in adults. Very few have specifically assessed the association between long-term air pollution exposure and lung function in the elderly. However, there is increasing evidence that the adverse effects of long-term exposure to air pollution are not limited to the developing lungs of children and that higher levels of air pollution are associated with a lower lung function and a faster decline in lung function in adults $[5,6,13-15]$. Also, certain people may be at increased risk, such as those with asthma or obesity, or those who are more genetically susceptible to the harmful effects of air pollution [16].

Evidence from longitudinal studies on beneficial effects of reductions in air pollution on lung function development is scarce. The Californian Children's Health Study (CHS) assessed the impact of changes in air pollution exposure on the developing lungs of children. In a small study of 110 children participating in the 
CHS, it has been shown that lung function growth is larger in children who moved to an area with lower levels of air pollution compared to children who moved to an area with higher levels of air pollution [17]. A more recent study within three cohorts of the CHS $(n=2120)$ showed that improvements in air quality from 1994 to 2011 in southern California were associated with measurable improvements in lung function growth from age 11 to 15 years and significant reductions in the proportion of children with a clinically

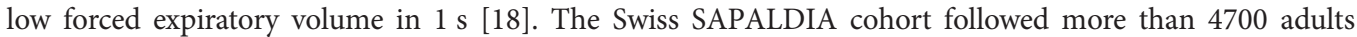
aged $42 \pm 11$ years over a period of 11 years and found that reductions in air pollution levels during that period were associated with a slower decline in lung function [19]. The study by HüLs et al. [20] from the German SALIA cohort that has been published in this month's issue of the European Respiratory Journal is a welcome addition to the currently limited evidence on the beneficial effects of reductions in air pollution exposure on the aging lungs. HüLs et al. [20] followed 601 women aged 55 years living in North Rhine-Westphalia, including the Ruhr area, one of Europe's largest industrial areas, over a period of 30 years during which air pollution levels decreased drastically. They linked data on lung function from three follow-ups to changes in air pollution levels during that 30-year period and found that reductions in air pollution levels, in particular levels of nitrogen oxides and PM10, were associated with a relative improvement in lung function [20]. These findings together with the findings of previous studies suggest that our lungs benefit from reduced air pollution exposure across the entire lifespan. The authors also addressed the important question of who benefits most from the reduction of air pollution and investigated potential effect modifications by body mass index (BMI) and genetic susceptibility to low lung function. Whereas no interactions with BMI were found, the authors report that subjects with a high genetic risk of having low lung function benefitted less from the improvements in air pollution than those with a low genetic risk.

There are several methodological challenges when studying health effects of air pollution in the elderly. Firstly, attrition, especially from mortality and disability, is a concern in longitudinal studies of older persons as a greater physical disability is associated with an increased likelihood of being lost to follow-up [21]. If the risk factor of interest also influences attrition, which can be assumed to be the case for air pollution, the resulting differential attrition can cause bias in the estimated association between that factor and lung function decline [22], as for example those who survive may have other beneficial characteristics (e.g. genetic background) that protect them and their lungs from the adverse effects of air pollution. In their study, Hüls et al. [20] did not perform a formal analysis of the potential impact of selective attrition on the estimated relationship between improved air quality and lung function of the elderly. However, they acknowledge that loss-to follow-up was selective and that amongst others, women with higher exposures to air pollution and worse respiratory health were more often lost to follow-up, which may have biased their findings. Secondly, loss to follow-up resulted in a study sample that was about $22 \%(601 / 2785)$ of the original study population. When combining this with genetic data, this number dropped to 462. This sample size is rather limited for genetic studies, and none of the genetic variants were significantly associated with lung function. However, rather than studying the genetic variants one by one, a weighted genetic risk score was calculated based on the number and strength of the risk variants from a previous large genome-wide association study [23]. Using this risk score, the authors showed that the largest benefits of the reduction in air pollution were observed in women with a low genetic risk score. This interesting observation adds to the currently limited evidence about the potential modifying role of genetics in the association between improved air quality and lung function decline in adults from the SAPALDIA cohort that suggests that, in particular, cell cycle control genes may be involved [24]. However, further investigation is warranted of how genetic and environmental factors interact in causing low lung function in the elderly, possibly resulting in COPD, leading to identification of susceptible subgroups, and larger studies in both sexes, as well as in patients with asthma or cardiovascular disease, are needed. Thirdly, the assessment of air pollution over a 30 -year period is challenging and exposure measurement error is a concern. The authors estimated individual exposures at the home addresses with a purely spatial land-use regression model that is based on an air pollution measurement campaign performed in 2008-2009. They then back-extrapolated and forecasted estimated exposures to the time points of the medical examinations using the temporal trend measured at a single routine air quality monitor in the study area assuming spatial contrasts being stable over the 30-year period study, which is a rather strong assumption.

In conclusion, the study by HüLs et al. [20] illustrates that the benefits of improved air quality extend across the life span into the elderly population and that the fetal period and childhood, although critical for the development of lung function, are thus not the only windows of opportunity for preventing the adverse effects of air pollution. This is an important finding as in 2017, one-fifth of the population of the European Union was 65 years or older [25] and this number is expected to increase in the coming years. Clean air contributes to healthy ageing, benefitting the lungs of the ageing population. 
Conflict of interest: U. Gehring reports institutional grants from Lung Foundation of the Netherlands, and Netherlands Organization for Scientific Research (NWO), outside the submitted work. G. Koppelman reports institutional grants from Lung Foundation of the Netherlands, Ubbo Emmius Foundation, TETRI Foundation and TEVA, the Netherlands, outside the submitted work.

\section{References}

1 Lim SS, Vos T, Flaxman AD, et al. A comparative risk assessment of burden of disease and injury attributable to 67 risk factors and risk factor clusters in 21 regions, 1990-2010: a systematic analysis for the Global Burden of Disease Study 2010. Lancet 2012; 380: 2224-2260.

2 Cohen AJ, Brauer M, Burnett R, et al. Estimates and 25-year trends of the global burden of disease attributable to ambient air pollution: an analysis of data from the Global Burden of Diseases Study 2015. Lancet 2017; 389: 1907-1918.

3 Brunekreef B, Holgate ST. Air pollution and health. Lancet 2002; 360: 1233-1242.

4 Sin DD, Wu L, Man SF. The relationship between reduced lung function and cardiovascular mortality: a population-based study and a systematic review of the literature. Chest 2005; 127: 1952-1959.

5 Gotschi T, Heinrich J, Sunyer J, et al. Long-term effects of ambient air pollution on lung function: a review. Epidemiology 2008; 19: 690-701.

6 Health Effects Institute Panel on the Health Effects of Traffic-Related Air Pollution. Traffic-Related Air Pollution: A Critical Review of the Literature on Emissions, Exposure, and Health Effects. Health Effects Institute, Boston, 2010.

7 Schultz ES, Litonjua AA, Melen E. Effects of long-term exposure to traffic-related air pollution on lung function in children. Curr Allergy Asthma Rep 2017; 17: 41

8 Milanzi EB, Koppelman GH, Smit HA, et al. Air pollution exposure and lung function until age 16 years: the PIAMA birth cohort study. Eur Respir J 2018; 52: 1800218.

9 Schultz ES, Gruzieva O, Bellander T, et al. Traffic-related air pollution and lung function in children at 8 years of age - a birth cohort study. Am J Respir Crit Care Med 2012; 186: 1286-1291.

10 Gehring U, Gruzieva O, Agius RM, et al. Air pollution exposure and lung function in children: the ESCAPE project. Environ Health Perspect 2013; 121: 1357-1364.

11 Rice MB, Rifas-Shiman SL, Litonjua AA, et al. Lifetime exposure to ambient pollution and lung function in children. Am J Respir Crit Care Med 2016; 193: 881-888.

12 Stern DA, Morgan WJ, Wright AL, et al. Poor airway function in early infancy and lung function by age 22 years: a non-selective longitudinal cohort study. Lancet 2007; 370: 758-764.

13 Lepeule J, Litonjua AA, Coull B, et al. Long-term effects of traffic particles on lung function decline in the elderly. Am J Respir Crit Care Med 2014; 190: 542-548.

14 Adam M, Schikowski T, Carsin AE, et al. Adult lung function and long-term air pollution exposure. ESCAPE: a multicentre cohort study and meta-analysis. Eur Respir J 2015; 45: 38-50.

15 Rice MB, Ljungman PL, Wilker EH, et al. Long-term exposure to traffic emissions and fine particulate matter and lung function decline in the Framingham heart study. Am J Respir Crit Care Med 2015; 191: 656-664.

16 Gref A, Merid SK, Gruzieva O, et al. Genome-wide interaction analysis of air pollution exposure and childhood asthma with functional follow-up. Am J Respir Crit Care Med 2017; 195: 1373-1383.

17 Avol EL, Gauderman WJ, Tan SM, et al. Respiratory effects of relocating to areas of differing air pollution levels. Am J Respir Crit Care Med 2001; 164: 2067-2072.

18 Gauderman WJ, Urman R, Avol E, et al. Association of improved air quality with lung development in children. N Engl J Med 2015; 372: 905-913.

19 Downs SH, Schindler C, Liu LJ, et al. Reduced exposure to PM10 and attenuated age-related decline in lung function. N Engl J Med 2007; 357: 2338-2347.

20 Hüls A, Sugiri D, Abramson MJ, et al. Benefits of improved air quality on ageing lungs: impacts of genetics and obesity. Eur Respir J 2019; 53: 1801780.

21 Chatfield MD, Brayne CE, Matthews FE. A systematic literature review of attrition between waves in longitudinal studies in the elderly shows a consistent pattern of dropout between differing studies. J Clin Epidemiol 2005; 58: $13-19$.

22 Weuve J, Tchetgen Tchetgen EJ, Glymour MM, et al. Accounting for bias due to selective attrition: the example of smoking and cognitive decline. Epidemiology 2012; 23: 119-128.

23 Soler Artigas M, Wain LV, Miller S, et al. Sixteen new lung function signals identified through 1000 Genomes Project reference panel imputation. Nat Comm 2015; 6: 8658.

24 Imboden M, Schwartz J, Schindler C, et al. Decreased PM10 exposure attenuates age-related lung function decline: genetic variants in p53, p21, and CCND1 modify this effect. Environ Health Persp 2009; 117: 1420-1427.

25 Eurostat. Population Structure and Ageing. https://ec.europa.eu/eurostat/statistics-explained/index.php/Population structure_and_ageing Date last accessed: February 20, 2019. 